\title{
Risk sensitive optimal control framework applied to delay tolerant networks
}

\author{
Eitan Altman*, Veeraruna Kavitha*+, Francesco De Pellegrini ${ }^{\dagger}$, Vijay Kamble ${ }^{\Uparrow}$ and Vivek Borkar ${ }^{\ddagger}$ \\ *Maestro group, INRIA, Sophia Antipolis, France. eitan.altman@inria.fr～+ LIA, University of Avignon, France. kavitha.voleti_veeraruna@inria.fr \\ ${ }^{\dagger}$ CREATE-NET, Trento, Italy. francesco.depellegrini@create-net.org $\mathbb{1}$ EECS Dept, UC Berkeley. vjk@eecs.berkeley.edu \\ ${ }^{\ddagger}$ Tata Institute of Fundamental Research, Mumbai, INDIA. borkar@tifr.res.in
}

\begin{abstract}
Epidemics dynamics can describe the dissemination of information in delay tolerant networks, in peer to peer networks and in content delivery networks. The control of such dynamics has thus gained a central role in all of these areas. However, a major difficulty in this context is that the objective functions to be optimized are often not additive in time but are rather multiplicative. The classical objective function in DTNs, i.e., the successful delivery probability of a message within a given deadline, falls precisely in this category, because it takes often the form of the expectation of the exponent of some integral cost. So far, models involving such costs have been solved by interchanging the order of expectation and the exponential function. While reducing the problem to a standard optimal control problem, this interchange is only tight in the mean field limit obtained as the population tends to infinity.

In this paper we identify a general framework from optimal control in finance, known as risk sensitive control, which let us handle the original (multiplicative) cost and obtain solutions to several novel control problems in DTNs. In particular, we can derive the structure of state-dependent controls that optimize transmission power at the source node. Further, we can account for the propagation loss factor of the wireless medium while obtaining these controls, and, finally, we address power control at the destination node, resulting in a novel threshold optimal activation policy. Combined optimal power control at source and destination nodes is also obtained.
\end{abstract}

Index Terms-Delay Tolerant Networks, Markov Decision Process, Risk Sensitive Control

\section{INTRODUCTION}

Delay Tolerant Networks (DTNs) gained the interest of the research community in recent past [2], [3]. They have been identified as a promising mean to transport data in intermittently connected networks. DTNs in particular, sustain communications in a networked system where no continuous connectivity guarantee can be assumed [4], [5]. Messages are carried from source to destination via relay nodes adopting store and carry type forwarding protocols; such protocols basically rely on the underlying node mobility pattern. The core problem in DTNs is to efficiently route messages towards the intended destination. We observe that traditional techniques for routing perform very poorly in this context due to frequent disruptions, and furthermore mobile nodes rarely possess information on the upcoming encounters they are going to experience [6], [7]. An intuitive and rather robust solution is to disseminate multiple copies of the message in the network. This is meant to ensure that at least some of them will reach the destination node within some deadline [5], [8].
The above scheme is referred as epidemic-style forwarding [9], which is similar to the spread of infectious diseases. Each time a message-carrying node encounters a node without message, infects it by passing on a copy. Finally, the destination receives the message when it meets an infected node. As in biology, DTNs literature refers to contacts as those events when the message can be forwarded.

In this paper, we confine our analysis to the two hop routing protocol. This choice is dictated both by efficiency reasons and by the possibility to implement all the forwarding control on board of the source node. In fact, under the two hop routing protocol, the source transmits copies of its message to all mobiles it encounters. A relay, conversely, forwards the message copy it has to the destination only [10].

Optimizing the performance of DTNs requires to maximize the successful delivery probability of a message within a given deadline. However, in order to do so, one has to trade off system resources to increase the success rate.

A natural assumption is that both source and destination nodes aim at maximizing the delivery success probability. But, they have their own individual constraints on their resources (e.g., power) both of which are pivotal in influencing this delivery probability. So, we consider a joint optimization problem with soft constraint on the power of both the source and the destination. At the source, we consider two types of power control: 1) controlling power per transmission which in turn effects the transmission range and hence the contact rate; 2) controlling the number of copies delivered to the contacted mobiles while keeping the power per transmission fixed. The destination only controls the power per transmission.

For power control of the first type, our model accounts also for the influence of pathloss factor of the wireless medium.

The technique used in this paper helps in obtaining the complete characterization of the optimal control for some of the aforementioned control problems while for the others, interesting analytical properties are derived. In particular, the technique adopted here is a general framework from optimal control in finance, known as risk sensitive control, which deals effectively with the multiplicative costs involved in this context. Below, we introduce the fundamental structure of this general optimization problem. 


\section{Delay Tolerant Networks and Risk Sensitive MDP}

Consider a network with a set of $I$ communities. A community $i$ consists of a time varying number of members which we denote by $X_{i}(t)$. In the context of DTNs, $X_{i}$ usually denotes the number of infected nodes within the $i$-th community. Now, consider some tagged node which we shall call the "destination". We assume that each member of community $i$ has a Poisson contact process with the destination at rate $\nu_{i}$. Conditioning on the processes $X_{i}(t)$, these Poisson processes are assumed to be independent, and thus the destination receives messages carried by members of the whole network at a time varying rate of $\sum_{i=1}^{I} X_{i}(t) \nu_{i}$. The probability that it receives no messages during $[s, t]$ is (see equation (3), [8])

$$
\begin{aligned}
P\left(\text { no reception } \mid X_{i}(r), r\right. & \in[s, t], i=1, \ldots, I) \\
& =\exp \left(-\int_{s}^{t} \sum_{i} \nu_{i} X_{i}(r) d r\right)
\end{aligned}
$$

Unconditioning, we get

$$
\begin{aligned}
P_{f} & :=P(\text { no reception })=E\left[\exp \left(-\nu \int_{s}^{t} c(r) d r\right)\right] \\
c(r) & =\sum_{i} \frac{\nu_{i}}{|\nu|} X_{i}(r)
\end{aligned}
$$

where $\nu \neq 0$ is some appropriate constant and $E$ denotes the expectation operator. If $X_{i}(t)$ are piecewise constant over the intervals $[n \Delta,(n+1) \Delta)$ then the integral in the exponent can be replaced by a summation over time: this is precisely the case considered in the rest of the paper.

Now assume that $X_{i}(t)$ is the function of some controlled Markov decision process $Y(t), \lambda(t)$ where $Y$ is the state of the process and $\lambda(t)$ is the control. Then the expression $P_{f}$ is a standard object in the theory of controlled Markov chains: this is the so called "risk sensitive cost criterion", which has had many applications in financial mathematics. The cost function we need to optimize in the paper, will actually be of the form (1) with $\nu>0$; risk-sensitive control literature in economics classify this case as risk avert. ${ }^{1}$

In previous delay tolerant literature [8], [11], one changes the order of expectation and exponent and is thus faced with an optimization of the exponential function of the expected integral. By Jensen's inequality, this is equivalent to optimizing a bound over the original function. Nevertheless, this bound has been shown to be tight in several contexts as the population size grows. Thus, in the mean field limit, it is still possible to overcome the optimization of the expectation of the exponential of the integral [12]. The technique shown in this paper

\footnotetext{
${ }^{1}$ Given a probability distribution of a nonnegative payoff, an agent is said to be non-sensitive to risk, if the agent has no preference between receiving that payoff or receiving its expectation. This case results in the limit $\nu \rightarrow 0$ in (1) (actually logarithm of the cost in (1) divided by $\nu$ ), which converges to the original (additive cost) optimal control problem. A risk seeking agent, the case with $\nu<0$, prefers receiving the random variable over its expectation, and a risk avert agent, case with $\nu>0$, prefers always the expectation.
}

works without this exchange of expectation and exponent and hence works even in the case of finite populations.

What is common to the standard interpretation of a risk sensitive cost and to the one we have in our case? The risk sensitive cost criterion accounts for the time correlation of the integrand. In contrast, the risk neutral bound obtained by optimizing the expectation of the exponent is only a function of the marginal distribution of the random variables.

There is a wealth of tools to solve control problems with risk sensitive cost. In general one finds the same tools as in standard additive cost MDPs but they take instead, a multiplicative form as seen later. Thus a Bellman equation exists as well, and under suitable conditions the controls that optimize the corresponding dynamic programming operator at a given state define the optimal policy. As in standard MDPs, we can use value iteration under a multiplicative form [13]) to compute the optimal value and policy for finite horizon cost.

In literature, heterogeneous DTNs with multiple communities, i.e., several classes of nodes, are a current interest of the research [14]. The tools we introduce throughout this work fit the general case (1); however, within the scope of this paper, we shall consider a single community DTN example.

\section{SySTEM MODEL}

Consider a large area with $N$ active DTN mobiles and assume that full connectivity is not guaranteed at each point in time. We consider a static source and destination: the communication between the two has to happen only with the help of the active mobiles. Source and destination can transmit/receive only with mobiles that are within their radio range. The radio range depends upon the power used by the transmitter per transmission. The mobiles are wandering freely in the area. A contact is said to happen with the source/destination whenever a mobile enters the radio range of the source/destination.

When the area is large and transmission range is small, this contact process is a rare phenomenon and is modeled by a Poisson process, i.e., the time to contact is exponentially distributed ( [15]). The validity of this model has been discussed in [15], and its accuracy has been shown for a number of mobility models (Random Walker, Random Direction, Random Waypoint). We further assume that each contact duration is sufficient to fully transfer the message.

Also, every message generated at the source has to be delivered to the destination within a given deadline $T$.

The source passes on the copy of the message to some of the contacted mobiles, taking into consideration its own resources. Recall that since we consider the two hop protocol, infected mobiles can only deliver the message to the destination. We say a delivery failed if none of the infected mobiles come in contact with the destination before the deadline $T$. The source has to spend its power for two purposes: 1) it has to continuously show its presence (done with help of beaconing), 2) it has to deliver the message to the contacted mobiles using a wireless link, which requires positive transmission power. 
In any case, these devices are power limited and hence have constraint on the total power that can be used.

The more power the device uses per transmission the bigger is its transmission range and hence larger will be the rate with which the mobiles can contact. This identifies a clear trade off for active time and power in the system. In fact, larger power per transmission causes more mobiles infected per unit time but for a shorter duration, while lower power per transmission causes lesser number of mobiles infected per unit time but for longer duration. Thus the source has to use an optimal power strategy before the deadline $T$ in order to to minimize the probability of delivery failure. Also, the transmission range depends upon the propagation coefficient of the wireless medium. Finally, the optimal power strategy depends both upon the total power constraint and the propagation coefficient.

Unlike the source, the destination receives the message only once and hence it spends most of its power to signal its presence (i.e., for beaconing). In some cases it might wish to receive more copies of the messages, due to the possible communication errors. Even in this case, the number of copies received at the destination is much smaller than the number of mobiles infected by the source, as the reception is possible only after the joint occurrence of two rare events. Hence, in all cases, the destination mostly spends its power for signaling.

The source and destination have a common goal, to minimize the delivery failure probability. This they have to do, under their own power constraints. We obtain optimal policies for both the source and destination which minimize the power spent and maximize the probability of successful delivery.

The rate of contacts, $(\lambda, \gamma$ respectively for source-mobile, destination-mobile contacts), depend upon the speed of the mobiles, propagation characteristics of the wireless medium, the power per transmission at which the source/destination is operating [10], [15]. In Appendix B we relate the power spent to sustain a given contact rate and the attenuation coefficient.

We consider a time slotted system and the goal is to design $\left\{\lambda_{k}, \gamma_{k}\right\}_{k}$ optimally. Let $X_{k}$ denote the number of infected mobiles at the beginning of time slot $k$, where time slots have unit duration without loss of generality. This represents the state of the system. Let $I_{k}$ represent the number of nodes infected in slot $k$. Then, $X_{k+1}=X_{k}+I_{k}$ where with $q_{\lambda}:=$ $e^{-\lambda}$ (probability of no contact in one time slot)

$$
\begin{aligned}
p_{n_{1}, n_{1}+n_{2}}^{\lambda} & :=P\left(I_{k}=n_{2} \mid X_{k}=n_{1}\right)=p_{n_{2}}^{\lambda}\left(N-n_{1}\right) \text { with } \\
p_{n_{2}}^{\lambda}(r) & :=\left(\begin{array}{c}
r \\
n_{2}
\end{array}\right)\left(1-q_{\lambda}\right)^{n_{2}} q_{\lambda}^{r-n_{2}} \text { for all } n_{2} \leq N-n_{1},
\end{aligned}
$$

represents the control-dependent transition probabilities.

Now, assume a message is not delivered within the deadline $T$. Then it is "not delivered" by any one of the $X_{t}$ infected mobiles present at the beginning of the slot, during the slot $t$ and if this "no delivery" happens in all the $T$ slots. Conditioned on the state trajectory, the events in various slots are independent and thus the overall delivery failure probability is the product of the delivery failure probability in each slot. The unconditioned delivery failure probability will be the expected value of this product of slot failure probabilities. Thus the probability of the message not reaching the destination within the time deadline $T$, for a given sequence of contact time parameters $\Lambda:=\left\{\lambda_{t}\right\}_{0 \leq t \leq T-1}, \Upsilon:=\left\{\nu_{t}\right\}_{0 \leq t \leq T-1}$ is

$$
P_{f}(\Lambda, \Upsilon)=E\left[e^{-\sum_{t} \nu_{t} X_{t}}\right]
$$

Aim is to choose $\Lambda \in \Omega_{S}^{T}, \Upsilon \in \Omega_{D}^{T}$ with $\Omega_{S}:=$ $\left\{0, \lambda_{1}, \ldots, \lambda_{M_{S}}\right\} \Omega_{D}:=\left\{0, \nu_{1}, \ldots, \nu_{M_{D}}\right\}$ in a optimal way so as to minimize the failure probability $P_{f}$ along side minimizing the power consumption at both the source and destination. We further assume that $\lambda^{i} \leq \lambda^{j}$ for $i \leq j$.

The power constraint can be modeled in two ways:

Soft Power Constraint. In some applications, there is no hard constraint on the power spent, but rather one needs to minimize the total power spent $p$. In this case we have two contrasting costs a) the cost for spending the power represented by a function $c(p)$ or equivalently $\left.e^{c(p)}, \mathrm{b}\right)$ the cost if the message has not been delivered in terms of $P_{f}$.

In order to give fair importance to both the costs, proportional fairness is attained by minimizing:

$$
E\left[P_{f} e^{h c(p)}\right]=E\left[e^{-\sum_{t} \nu_{t} X_{t}+h c(p)}\right],
$$

where $h$ is the weight given to the power cost.

Hard Power Constraint. Some applications have hard constraint on the total power spent: one needs to minimize the failure probability under power constraint (for some $B<\infty$ ):

$$
\min E\left[e^{-\sum_{t} \nu_{t} X_{t}}\right] \text { subject to } c(p) \leq B \text {. }
$$

The current paper works under the soft constraint. We consider two types of power costs $c(p)$ in the following and consider the resulting source and or destination controls. We end this section by introducing the two types of pure source controls, i.e., the case when destination rate $\nu$ is constant. The other controls, namely pure destination and combined source-destination controls, are introduced incrementally in the sections after pure source control.

Pure source Delivery Control. The source does not change the power per transmission but rather optimizes the total power spent by controlling the number of message copies. Thus the transmission range remains constant and hence the exponential contact rate remains a constant, namely $\lambda$. However, when the source comes in contact with a mobile, it delivers the message with probability $q_{t} \in[0,1]$. In turn, the effective contact rate is $\lambda_{t}=\lambda q_{t}$. This case is appropriate whenever the source uses some smart techniques to spend minimal power for beaconing while most of it's power is utilized only for delivery of copies of the message. One such example is when the mobiles themselves detect the existence of the source and wake up the otherwise sleeping source. Overall, the power spent by the source depends directly upon the total number of infected mobiles, $X_{T}$, at the end of the delivery deadline and 
thus $c(p) \propto X_{T}$. The control problem writes:

$$
\min _{\Lambda \in \Omega_{S}^{T}} E\left[e^{-\nu \sum_{t} X_{t}+h X_{T}}\right]
$$

Pure source Power Control. The source does not control the number of message deliveries but rather controls the power per transmission. The transmission range varies, depending upon the path loss coefficient, with the power per transmission; this in turn varies the mean contact time, $1 / \lambda$ (see Appendix B). With this control, the power is also used for maintaining the transmitting range high/low throughout and hence the beaconing capabilities also vary from slot to slot. This case is appropriate for the situations in which the source has to spend significant power for both beaconing purposes as well as for delivery of message copies. In this case, the power is inversely proportional to the contact rates (proportionality coefficient depends on path loss coefficient) and hence consider the following minimization ${ }^{2}$

$$
\min _{\Lambda \in \Omega_{S}^{T}} E\left[e^{-\nu \sum_{i} X_{i}+h \sum_{i}\left(\lambda_{i}\right)^{\beta}}\right] .
$$

In the next sections we detail the above control problems and derive, solve for each case the corresponding risk sensitive dynamic programming equations ( [16]).

\section{Pure Source Controls}

In this section, we obtain complete characterization of the optimal delivery control and some interesting properties of the optimal power control, when destination maintains it's power constant (and hence its contact rate remains constant at $\nu$ ). We also obtain complete characterization of the optimal power control for the special case of hard controls, i.e., the controls for the case with only two possible actions $\Omega_{S}=$ $\left\{0, \lambda_{1}\right\}$. Results are obtained using the risk-sensitive dynamic programming (Risk-MDP) equations [16] under perfect state information. Observe that the state of the system, i.e. number of infected customers $X_{k}$, is known at the source.

\section{A. Delivery control}

The delivery control problem (2) results in the following Risk-MDP equations, for every $n$ :

$$
\begin{aligned}
& u^{T}(n)=e^{-\nu n+h n} \\
& u^{t}(n)=\min _{\lambda \in \Omega_{S}}\left(e^{-\nu n} \sum_{n \leq n^{\prime} \leq N} p_{n, n^{\prime}}^{\lambda} u^{t+1}\left(n^{\prime}\right)\right) \\
& \lambda_{t}^{*}(n)=\underset{\lambda \in \Omega_{S}}{\operatorname{argmin}}\left(e^{-\nu n} \sum_{n \leq n^{\prime} \leq N} p_{n, n^{\prime}}^{\lambda} u^{t+1}\left(n^{\prime}\right)\right)
\end{aligned}
$$

Here, $\lambda_{t}^{*}(n)$ represents the optimal control at time $t$ if the state $X_{t}$ was at $n$. The optimal cost and control for any given initial condition $X_{0}$ are obtained by solving the above Risk-MDP equations using backward recursions. Starting with $t=T-1$

\footnotetext{
${ }^{2}$ where, as explained in Appendix B, $\beta=\alpha$ or $\alpha / 2$, with $\alpha$ the loss factor.
}

one obtains $u^{t}(n), \lambda_{t}^{*}(n)$ for all possible states $0 \leq n \leq N$ and all possible times $T \geq t \geq 0$. The minimum cost can now be read as $u^{1}\left(X_{0}\right)$ while the optimal control given by the sequence, $\lambda_{1}^{*}\left(X_{0}\right), \lambda_{2}^{*}\left(X_{1}^{*}\right) \cdots \lambda_{T}^{*}\left(X_{T-1}^{*}\right)$, where $X_{k}^{*}$ is the optimal state trajectory obtained via forward recursion after replacing at each step, $\lambda_{t}$ with $\lambda_{t}^{*}\left(X_{t-1}^{*}\right)$. Solving the MDP equations recursively we obtain (proof in Appendix A)

Lemma 1: Define, $t_{D c}^{*}:=\inf _{t \leq T}\{(T-t) \nu \leq h\}$. Then,

$\lambda_{t}^{*}(n)=0, \quad u^{t}(n)=e^{-(T-t+1) \nu n+h n}$ if $t \geq t_{D c}^{*}$ and

$\lambda_{t}^{*}(n)=\lambda_{M} \quad$ if $t<t_{D c}^{*} \quad$ for all $n$.

Thus the optimal delivery control is a threshold control (deliver with maximum probability till threshold $t_{D c}^{*}$ and stop it completely afterwards). The interesting feature here is that the threshold is independent of the state $n$ and the strength of the active population $N$. It only depends upon $h$, which represents the weight given to the power cost, $\nu$ the contact rate for the destination and $T$ the delivery deadline. Larger deadlines $(T)$, larger destination contact rates $(\nu)$ increase the threshold $t_{D c}^{*}$. These two factors give more weight to the delivery probability cost and hence demand to deliver the message copies for larger durations. On the other hand, with larger $h$ threshold $t_{D c}^{*}$ decreases because a larger weight is given to power expenditure.

\section{B. Power control}

The power control (3) will result in the following Risk-MDP equations ( [16]) for any state $n$ :

$$
\begin{aligned}
& u^{T}(n)=e^{-\nu n} \\
& u^{t}(n)=\min _{\lambda \in \Omega_{S}}\left(e^{-\nu n+h \lambda^{\beta}} \sum_{n \leq n^{\prime} \leq N} p_{n, n^{\prime}}^{\lambda} u^{t+1}\left(n^{\prime}\right)\right) \\
& \lambda_{t}^{*}(n)=\underset{\lambda \in \Omega_{S}}{\operatorname{argmin}}\left(e^{-\nu n+h \lambda^{\beta}} \sum_{n \leq n^{\prime} \leq N} p_{n, n^{\prime}}^{\lambda} u^{t+1}\left(n^{\prime}\right)\right)
\end{aligned}
$$

Again, the optimal control can be computed as done in the previous section, as reported in Appendix A:

$$
\sum_{n \leq n^{\prime} \leq N} p_{n, n^{\prime}}^{\lambda} u^{T}\left(n^{\prime}\right)=e^{-\nu n}\left(e^{-\nu}+q_{\lambda}\left(1-e^{-\nu}\right)\right)^{N-n}
$$

Clearly at $N=n, \lambda^{*}(T-1, N)=0$. For $n<N$,

$u^{T-1}(n)=e^{-\nu(n+N)}\left[\min _{\lambda \in \Omega}\left(e^{Q_{n} \lambda^{\beta}}\left[P_{T-1} q_{\lambda}+1\right]\right)\right]^{N-n}$

where $Q_{n}:=\frac{h}{N-n}$ and $P_{T-1}:=e^{\nu}-1$. In this case we could only obtain a partial characterization of the control (proof in Appendix A and recall $\Omega_{S}=\left\{0, \lambda_{1}, \cdots, \lambda_{M_{D}}\right\}$ ):

Lemma 2: For all $n \geq n_{o f f}(\beta, h):=N-h \lambda_{1}^{\beta-1}$

$$
\lambda_{t}^{*}(n)=0 \text { and } u^{t}(n)=e^{-(T-t+1) n} \text { for all } t .
$$

For all $n<n_{o f f}(\beta, h)$,

$$
\lambda_{t}^{*}(n) \geq \lambda_{1} \text { for all } t .
$$


The following are consequences of the above lemma:

Switch off population : Immediately after the source infects $n_{\text {off }}(\beta, h)$ mobiles (which we refer as switch off population), it goes off to sleep mode. As $\beta$ increases, i.e., as the path loss factor of the wireless medium increases, the switch off population decreases. This is intuitive as with $\beta$ increasing more amount of power has to be spent for remaining on and or for delivering the message. Thus the power would be consumed more quickly.

Switch off/Sleep time : The above lemma only proves that there again exists a threshold beyond which the source does not deliver messages. In this control, it is done by not using any power for transmission. Hence, here the source actually goes to complete sleep mode after the threshold period. The threshold till which the source delivers is given by:

$$
t_{P c}^{*}=\inf _{t \leq T}\left\{X_{t}^{*}>n_{o f f}(\beta, h)\right\},
$$

where $X_{t}^{*}$ is the optimal state trajectory.

Need not be a threshold policy: For delivery control the optimal policy was threshold type, but here the optimal policy may not be threshold, i.e., it is possible that the optimal control (which is non zero till $t_{P c}^{*}$ ) can be time varying till $t_{P c}^{*}$.

More power for adverse conditions : Whenever $N \leq h \lambda_{1}^{\beta-1}$, the optimal control is to never switch on. This situation can arise either because the population is not large enough, or because the path loss factor $\beta$ is high or because the first non zero contact rate $\lambda_{1}$ is high. This trivial situation can be avoided by reducing the constraint on power, by reducing $h$.

Optimal control spreads over larger time frame and larger states if the first non zero contact rate $\lambda_{1}$ is small enough because as $\lambda_{1}$ decreases the switch off population $n_{o f f}$ increases to $N$. That is, when control is possible over finer grid, then the control spreads over larger time frame and for more states. Further, this spread increases with the increase in the path loss factor $\beta$ (as for $\lambda_{1}<1, \lambda_{1}^{\beta-1} \rightarrow 0$ as $\beta \rightarrow \infty$ ).

Finally, the following lemma provides complete characterization of the optimal risk-sensitive control for the case of two level control which is a direct consequence of the Lemma 2.

Lemma 3: When $\Omega_{S}=\left\{0, \lambda_{1}\right\}$ for all $t$,

$$
\lambda_{t}^{*}(n)=\left\{\begin{array}{lll}
0 & \text { if } & n \geq n_{o f f}(\beta, h) \\
\lambda_{1} & \text { if } & n<n_{o f f}(\beta, h) .
\end{array}\right.
$$

\section{Numerical examples:}

In general, since $Q_{n}$ is a function of the state, the relations that appear in the DP backward recursion at earlier stages do not provide a simple closed form. Numerical results can be obtained for a certain choice of the parameters. In all these results $u^{*}$ represents the optimal cost. We set $\Omega_{S}=$ $\left[0, \lambda_{M} / M, \cdots, \lambda_{M}\right]$ with $M=10$. In particular we are interested here in the impact of the coefficient $\beta$ since it expresses the cost paid by the source due to path loss factor on the optimal control. In Fig. 1 we observe the effect of such parameter for $\beta=1,2$. For $\beta=1$, there exists a thresholding effect both on the state and time. Conversely, for $\beta=2$, we
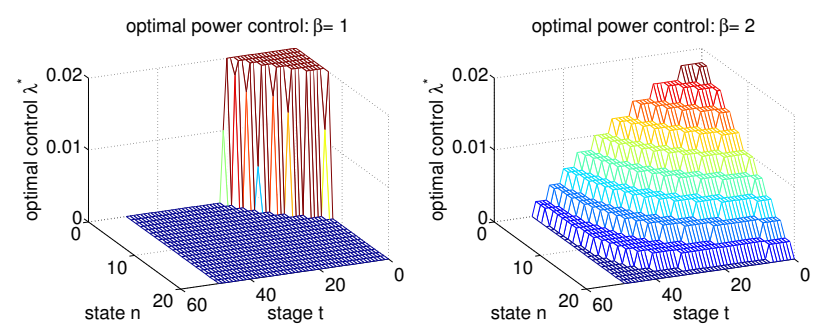

Fig. 1. Power control in the case of $\beta=1$ (left), $\beta=2$ (right); $\lambda_{M}=$ $2 \nu=2 \cdot 10^{-2} ; \log \left(u^{*}\right)=-1.53, M=10, N=20$, and $T=50$.

observe a rather smooth decrease of the optimal control. As given by Lemma 2 , for $h>N$ and $\beta=1$ the control vanishes.

As indicated by the analysis, higher values of the path loss coefficient force the control to become less and less concentrated at small values of the state, compared to the case $\beta=1$, i.e., to spread the control over larger time scales and larger number of states. It becomes more convenient to forward even when the number of infected nodes is high. However, this is done using a lower probability for smaller values of $n$. This kind of spread is seen only if the source can also operate at a very small but non zero contact rate (i.e., if $\lambda_{1}$ is small). On the other hand, if the source does not operate at sufficient number of levels of contact rates (as result $\lambda_{1}$ could be large), with larger values of $\beta$ the control can become trivial. Hence whenever the wireless medium experiences large propagation losses the source has to operate with higher granularity in the control space, i.e., it will require more smoother controls.

In Fig. 2 we first depicted the effect of the risk parameter $h$ on the forwarding control: it is apparent that larger penalties (larger $h$ ) force the control to switch off at earlier stages. In particular, for $h=N$ the control vanishes, i.e., no forwarding is performed at the source node.

Also, we depicted the impact of the path loss factor on the optimal control given a fixed optimal utility $\left(u^{*}\right)$; we emulated the generation of 1000 optimal control sample paths at the source node and compared the average optimal control corresponding to the same average utility. We maintained $u^{*}$ the same, by varying $h$ with $\beta$. We considered two different values of $u^{*}$ : smaller value, i.e., higher successful delivery probability, obtained using smaller penalty for power expenditure, i.e, smaller $h$, and larger one with larger penalty.

We observe in Fig. 2 that for small values of the penalty, the behavior of the controls is similar, and resembles a threshold type policy. However, for larger penalties - this has the meaning of increasing the weight of energy expenditure - the control for $\beta=1$ has still a threshold-like shape, whereas for larger values of the path loss factor, the decrease is gradual. Hence, even in time power spreading occurs, i.e., the source node is forced to reduce the forwarding probability, and to keep on forwarding over time. As in the case of threshold type policies, however, it is still convenient to spend energy as much as possible at the beginning of the interval, which explains the decreasing shape of the control. 

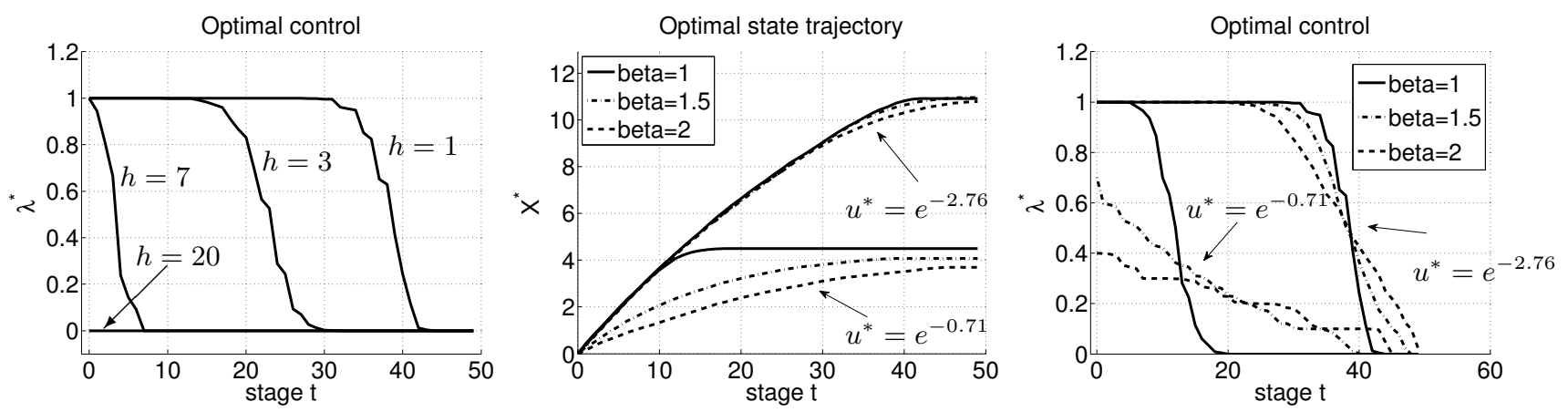

Fig. 2. Effect of $h$ on the optimal control for $\beta=1$ (left); Effect of $\beta$ : optimal state trajectory (center) and optimal control (right); settings as in Fig. 1, control values are normalized, reference utilities are superimposed.

\section{Pure Destination Controls}

In this section, we consider the pure destination controls: the source node contact happens at a constant exponential rate $\lambda$, whereas the destination operates power control. Recall that the destination cannot control the number of nodes infected and hence the only meaningful control in this case is power control. Thus we consider the following:

$$
\min _{\Upsilon \in \Omega_{D}^{T}} E\left[e^{-\sum_{t} \nu_{t} X_{t}+h \sum_{t} \nu_{t}^{\beta}}\right] .
$$

Recall that the message has to reach the destination before the end of time slot $T$. Here, we further assume that only the infected mobiles present at the beginning of the slot can reach destination during the slot. Thus, the destination has to control even at time slot $T$ (while the source can control only till slot $T-1$ ). Thus the corresponding Risk-MDP equations are:

$$
\begin{aligned}
u^{T+1}(n) & =1 \\
u^{t}(n) & =\min _{\nu \in \Omega_{D}}\left(e^{-\nu n+h \nu^{\beta}} \sum_{n \leq n^{\prime} \leq N} p_{n, n^{\prime}}^{\lambda} u^{t+1}\left(n^{\prime}\right)\right) \\
\nu_{t}^{*}(n) & =\underset{\nu \in \Omega_{D}}{\operatorname{argmin}}\left(e^{-\nu n+h \nu^{\beta}} \sum_{n \leq n^{\prime} \leq N} p_{n, n^{\prime}}^{\lambda} u^{t+1}\left(n^{\prime}\right)\right) .
\end{aligned}
$$

The evolution of state $X_{t}$ is independent of control and hence,

$$
u^{t}(n)=\min _{\nu \in \Omega_{D}}\left(e^{-\nu n+h \nu^{\beta}}\right) \sum_{n \leq n^{\prime} \leq N} p_{n, n^{\prime}}^{\lambda} u^{t+1}\left(n^{\prime}\right) \forall n .
$$

Thus, for all $t$ and $n$, the optimal control

$$
\begin{aligned}
\nu_{t}^{*}(n) & =g_{\nu}(n, \beta) \text { where } \\
g_{\nu}(n, \beta) & :=\arg \min _{\nu \in \Omega_{D}}\left(-\nu n+h \nu^{\beta}\right) .
\end{aligned}
$$

With $X_{t}$ representing the state trajectory, optimal policy, $\nu^{*}(t)=g_{\nu}\left(X_{t}, \beta\right)$. It is interesting to observe that even if the state evolution is independent of the control, the state still influences the system performance and hence the optimal policy depends upon the state value. However the optimal policy could be computed easily, because of the control independent state evolution. This policy is obtained assuming that the destination also has access to the current state, $X_{t}$, the number of infected mobiles. This assumption is satisfied ${ }^{3}$ if the source and destination have a very low rate link (back-haul kind), via which few control signals can be exchanged.

\section{A. State estimation}

When the destination does not have access to the full state information, it can estimate the channel state $X_{t}$ under the following conditions ${ }^{4}$ :

Large population limit: For large population, i.e., when $N$ is very large, using the strong law of large numbers, for almost all realizations,

$$
X_{t+1}=X_{t}+\sum_{j=1}^{N-X_{t}} \zeta_{j}(t) \approx X_{t}+\left(N-X_{t}\right)\left(1-e^{1-\lambda}\right),
$$

where $\zeta_{j}(t)$ is the indicator of the event that mobile $j$ meets the source at time $t$, whose probability $P\left(\left\{\zeta_{j}(t)=1\right\}\right)=1-e^{-\lambda}$. Continuous approximation: As the slot duration tends to zero (note $\lambda$ by our notations is a product of the actual contact rate and the slot duration and hence as $\lambda \rightarrow 0$ ), one can approximate the state dynamics with a solution of ODE

$$
\dot{X}=\lambda(N-X), \quad X(0)=0
$$

Thus the corresponding approximated optimal policy will be:

$$
\nu^{*}(t)=g_{\nu}\left(N-N e^{-\lambda t}, \beta\right) .
$$

\section{B. Switch on Threshold}

From (7), the optimal destination contact rate $\nu^{*}$ depends upon time $t$ only via the state at time $t$, i.e., it depends only upon the number of infected mobiles $n$. From (7), the optimal control increases with $n$ in the sense: $g_{\nu}(n, \beta)^{\beta-1} \leq n / h$. In

\footnotetext{
${ }^{3}$ Note that our solution assumes that state information is available. While this assumption may not be realistic in some applications, it does hold in hybrid networks where $3 \mathrm{G}$ or other network is used for signaling, while the data transfer uses a DTN contact based transfers. Such a scenario is reasonable when the transfered content is much larger than the signaling traffic.

${ }^{4}$ This situation can be better handled by partial information Risk MDP tools (for example, [1]), but this paper works only with full information case.
} 
fact, there exists a switch on population and a switch on time, reaching which the destination will be switched on:

$$
n_{\text {on }}(\beta, h):=\inf _{n}\left\{n>h \nu_{1}^{\beta-1}\right\}, t_{\text {Dest }}^{*}:=\inf _{t}\left\{X_{t} \geq n_{\text {on }}\right\} .
$$

The above is so because for all $n \geq n_{\text {on }},-n \nu_{1}+h \nu_{1}^{\beta}<0$ and hence $g_{\nu}(n, \beta) \geq \nu_{1}>0$. It is interesting to note that destination has a switch on threshold while the source has a switch off threshold for optimal policies. We will notice in the coming sections that even for combined source-destination control, we have same (w.r.t. to the number of infected mobiles) switch on threshold for the destination.

\section{Combined Source-Destination CONTROL}

The destination always performs power control while the source can either do the power control or the delivery control. Accordingly we have two different combined controls.

\section{A. Power Control at Source :}

The source and destination are far way and static. Hence they can be working in very different geographical locations and hence can experience different path losses. The objective function to be minimized in this case will be :

$$
\min _{\Lambda \in \Omega_{S}^{T}, \Upsilon \in \Omega_{D}^{T}} E\left[e^{-\sum_{t} \nu_{t} X_{t}+h_{d} \sum_{t} \nu_{t}^{\beta} d+h_{s} \sum_{t} \lambda_{t}^{\beta_{s}}}\right]
$$

Corresponding risk MDP equations for all $n$ and $t \leq T$ :

$$
\begin{aligned}
& u^{T+1}(n)=1 \\
& u^{t}(n)=\min _{\nu, \lambda}\left(e^{-\nu n+h_{d} \nu^{\beta} d+h_{s} \lambda^{\beta} d} \sum_{n \leq n^{\prime} \leq N} p_{n, n^{\prime}}^{\lambda} u^{t+1}\left(n^{\prime}\right)\right) \\
& \nu_{t}^{*}(n), \lambda_{t}^{*}(n) \\
& =\underset{\nu \in \Omega_{D}, \lambda \in \Omega_{S}}{\operatorname{argmin}}\left(e^{-\nu n+h_{d} \nu^{\beta} d+h_{s} \lambda^{\beta} d} \sum_{n \leq n^{\prime} \leq N} p_{n, n^{\prime}}^{\lambda} u^{t+1}\left(n^{\prime}\right)\right)
\end{aligned}
$$

The above optimization can be separated as before,

$$
\begin{aligned}
& u^{t}(n)= \min _{\nu \in \Omega_{D}}\left(e^{-\nu n+h_{d} \nu^{\beta_{d}}}\right) \\
& \min _{\lambda \in \Omega_{S}} e^{h_{s} \lambda^{\beta_{s}}} \sum_{n \leq n^{\prime} \leq N} p_{n, n^{\prime}}^{\lambda} u^{t+1}\left(n^{\prime}\right) \forall n .
\end{aligned}
$$

Thus $\nu_{t}^{*}(n)=g_{\nu}(n, \beta)$ where $g_{\nu}$ is defined in (7). That is, the destination optimal control will be same as that in pure destination control. The source control will however be different, as now, the cost in the new state will depend upon the new state also via the optimal destination control. The optimal destination control only depends upon state $n$ and not on time $t$ directly. Hence, it is possible to define,

$$
n_{\nu_{M_{D}}}:=\min _{0 \leq n \leq N}\left\{g_{\nu}\left(n^{\prime}, \beta\right)=\nu_{M_{D}} \text { for all } n^{\prime} \geq n\right\},
$$

which is the minimum number of infected mobiles for which the destination forever switches to the maximum contact rate, $\nu_{M_{D}}$. The number $n_{\nu_{M_{D}}}$ can easily be estimated as it is result of a simple optimization defined in (7). In the special case of hard controls, i.e., when $\Omega_{D}=\left\{0, \nu_{1}\right\}$,

$$
n_{\nu_{1}}=n_{o n}(\beta, h)=\min _{n}\left\{n \geq h_{d} \nu_{1}^{\beta_{d}-1}\right\} \text {. }
$$

Once the destination forever switches to its maximum rate, analysis will be similar to that in pure source control and thus,

Lemma 4: Let $n_{\text {off }}(\beta, h):=N-h_{s} \lambda_{1}^{\beta_{s}-1}$. For all $t$,

$\lambda_{t}^{*}(n)=0, u^{t}(n)=e^{-(T-t+1) n}$ if $n \geq \max \left\{n_{\nu_{M_{D}}}, n_{o f f}\right\}$

and $\lambda_{t}^{*}(n) \geq \lambda_{1}$ for all $t$ if $n_{\nu_{M_{D}}} \leq n<n_{o f f}(\beta, h)$.

Numerical Examples: Here we would like to provide insight into the behavior of the joint source-destination control. In Fig. 3 we reported on the the effect of the power control operated at the destination, i.e., based on its dependence on the parameter $h_{d}$, on the power control operated at the source node for $\beta=1$ and $h_{s}=1$. In Fig. 3 (left) we observe the optimal policy: the presence of a switch on policy for the destination discourages forwarding at later stages in the case when the number of infected nodes is small. Thus the control in this case is much different from what happens in the case $h_{d}=0$, where the threshold seen in over the companion graph in Fig. 1 is decreasing with the state for the same stage lag.

At larger states, there exists the opposite effect due to the cost of forwarding at the source, so that it is not worth to forward at too high rate when a large fraction of nodes have already been infected. Interestingly, the model predicts the presence of states, namely $n=5$, when the optimal control operates for much longer number of stages at maximum rate.

However, if we increase the cost at the destination, which in turn means that the destination switches on later, there exists a sudden increase of the cost function: as seen in Fig. 3 (center) and (right), once $h_{s}$ passes from 7 to 8 , the optimal control vanishes abruptly, showing a thresholding effect on the cost at the destination.

\section{B. Delivery Control at Source :}

The objective function to be minimized in this case is:

$$
\min _{\Lambda, \Upsilon} E\left[e^{-\sum_{t} \nu_{t} X_{t}+h_{d} \sum_{t} \nu_{t}^{\beta}+h_{s} X_{T}}\right]
$$

Corresponding risk sensitive DP equations for all $n$ are:

$$
\begin{aligned}
u^{T+1}(n) & =e^{h_{s} n} \\
u^{t}(n) & =\min _{\nu, \lambda}\left(e^{-\nu n+h_{d} \nu^{\beta}} \sum_{n \leq n^{\prime} \leq N} p_{n, n^{\prime}}^{\lambda} u^{t+1}\left(n^{\prime}\right)\right) \\
\nu_{t}^{*}(n), \lambda_{t}^{*}(n) & \\
& =\underset{\nu, \lambda}{\operatorname{argmin}}\left(e^{-\nu n+h_{d} \nu^{\beta}} \sum_{n \leq n^{\prime} \leq N} p_{n, n^{\prime}}^{\lambda} u^{t+1}\left(n^{\prime}\right)\right)
\end{aligned}
$$

The above optimization can be separated again and as before, the destination optimal control is same as that in pure destination control case, i.e., $\nu_{t}^{*}(n)=g_{\nu}(n, \beta)$. Further using similar logic as before, we have 

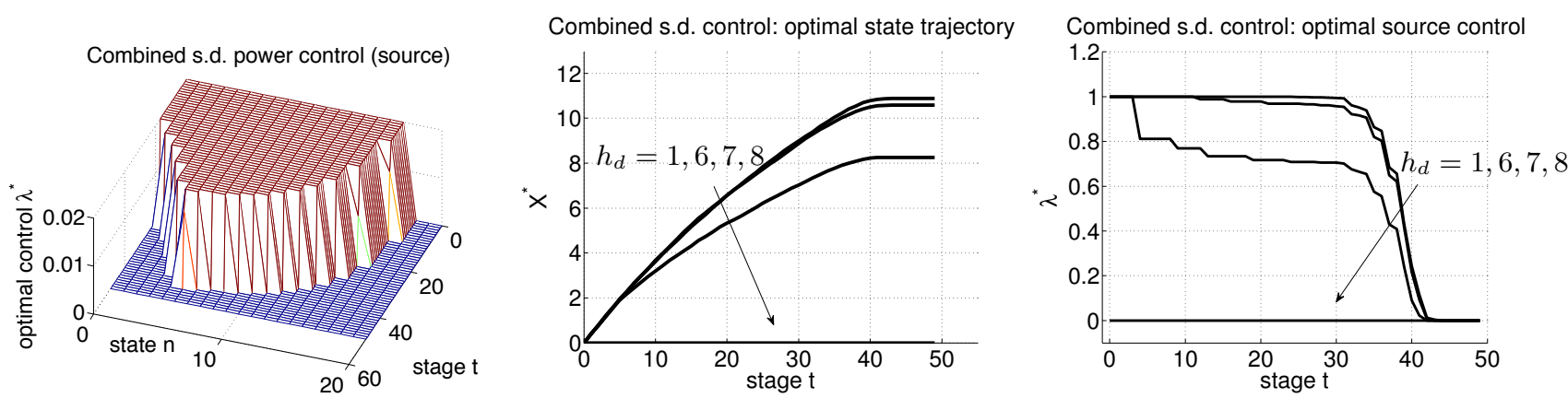

Fig. 3. Effect of $h_{d}$ on the optimal control for $\beta=1$ and $h_{s}=1$ : combined optimal control source destination at the source node (left), average optimal state trajectory (center), optimal source control policy (right); settings as in Fig. 1.

Lemma 5: For all $t \geq t_{D c}^{*}$ and for all $n \geq n_{\nu_{M_{D}}}$,

$$
\lambda_{t}^{*}(n)=0, \quad u^{t}(n)=e^{-(T-t+1) \nu_{M_{D}} n+h n},
$$

where $t_{D c}^{*}$ is as in Lemma 1 with $\nu$ replaced with $\nu_{M_{D}}$.

Acknowledgments: The first two author's work is supported by the Indo-French Centre for Promotion of Advanced Research (IFCPAR), project 4000-IT-1.

\section{CONCLUSiOnS}

In this paper we introduced a novel framework for the control of systems where the objective function has the form of a risk-sensitive cost. We applied such theory to DTNs, where delivery probability presents natively a risk-sensitive form. We provided several variants of the basic control problem where the success probability is traded off for power consumption in presence of propagation loss effects. We specialized the solution to the case when the control is performed at the source, at the destination or jointly. Compared to existing works, a whole set of new state-dependent closed loop policies provide here new insight in the control of DTNs.

\section{REFERENCES}

[1] M. R. James, J. Baras, and R. J. Elliott, Risk-sensitive control and dynamic games for partially observed discrete-time nonlinear systems, IEEE Trans. Automatic. Contr., vol. 39, pp. 780792, Apr. 1994.

[2] S. Burleigh, L. Torgerson, K. Fall, V. Cerf, B. Durst, K. Scott, and H. Weiss, "Delay-tolerant networking: an approach to interplanetary Internet," IEEE Comm. Magazine, vol. 41, pp. 128-136, June 2003.

[3] L. Pelusi, A. Passarella, and M. Conti, "Opportunistic networking: data forwarding in disconnected mobile ad hoc networks," IEEE Communications Magazine, vol. 44, no. 11, pp. 134-141, November 2006.

[4] A. Chaintreau, P. Hui, J. Crowcroft, C. Diot, R. Gass, and J. Scott, "Impact of human mobility on opportunistic forwarding algorithms," IEEE Transactions on Mobile Computing, vol. 6, pp. 606-620, 2007.

[5] T. Spyropoulos, K. Psounis, and C. Raghavendra, "Efficient routing in intermittently connected mobile networks: the multi-copy case," ACM/IEEE Transactions on Networking, vol. 16, pp. 77-90, Feb. 2008.

[6] M. M. B. Tariq, M. Ammar, and E. Zegura, "Message ferry route design for sparse ad hoc networks with mobile nodes," in Proc. of ACM MobiHoc, Florence, Italy, May 22-25, 2006, pp. 37-48.
[7] W. Zhao, M. Ammar, and E. Zegura, "Controlling the mobility of multiple data transport ferries in a delay-tolerant network," in Proc. of IEEE INFOCOM, Miami USA, March 13-17 2005.

[8] E. Altman, T. Başar, and F. De Pellegrini, "Optimal monotone forwarding policies in delay tolerant mobile ad-hoc networks," in Proc. of ACM/ICST Inter-Perf. Athens, Greece: ACM, October 242008.

[9] A. Vahdat and D. Becker, "Epidemic routing for partially connected ad hoc networks," Duke University, Tech. Rep. CS-2000-06, 2000.

[10] R. Groenevelt, P. Nain, and G. Koole, "The message delay in mobile ad hoc networks," Performance Evaluation, vol. 62, no. 1-4, pp. 210-228, October 2005

[11] E. Altman, G. Neglia, F. De Pellegrini, and D. Miorandi, "Decentralized stochastic control of delay tolerant networks," in Proc. of INFOCOxM, Rio de Janeiro, Brazil, April 19-25 2009.

[12] E. Altman, "Competition and cooperation between nodes in delay tolerant networks with two hop routing," in Proc. of Netcoop, Eindhoven, The Netherlands, November 2009.

[13] D. Jacobson, "Optimal stochastic linear systems with exponential criteria and their relation to differential games," IEEE Trans. Automat. Control, vol. 18, pp. 124-131, 1973.

[14] A. Chaintreau, J.-Y. L. Boudec, and N. Ristanovic, "The age of gossip: Spatial mean-field regime," in Proc. of ACM Sigmetrics, Seattle, June 2009.

[15] R. Groenevelt and P. Nain, "Message delay in MANETs," in Proc. of Sigmetrics. Banff, Canada: ACM, June 6 2005, pp. 412-413, see also R. Groenevelt, Stochastic Models for Mobile Ad Hoc Networks. PhD thesis, University of Nice-Sophia Antipolis, April 2005.

[16] S. Coraluppi and S. I. Marcus, "Risk-sensitive queueing," in Proc. 35th Annual Allerton Conf. on Communication, Control, and Computing, Urbana, IL, September 28-October 1.

\section{APPENDIX A}

Proof of Lemma 1: We start with time $t=T-1$. For this time, one can easily simplify the $\lambda$ dependent part of (5) as,

$$
\begin{aligned}
& \sum_{n \leq n^{\prime} \leq N} p_{n, n^{\prime}}^{\lambda} u^{T}\left(n^{\prime}\right) \\
& \quad=\sum_{0 \leq n^{\prime} \leq N-n}\left(\begin{array}{c}
N-n \\
n^{\prime}
\end{array}\right) q_{\lambda}^{N-n-n^{\prime}}\left(1-q_{\lambda}\right)^{n^{\prime}} e^{-(\nu-h)\left(n^{\prime}+n\right)} \\
& =e^{(-\nu+h) n}\left(q_{\lambda}+\left(1-q_{\lambda}\right) e^{-\nu+h}\right)^{N-n}
\end{aligned}
$$

Now if $\nu<h$ then, $e^{-\nu+h}>1$ and because of monotonicity of the function $\lambda \mapsto e^{-\nu+h}+q_{\lambda}\left(1-e^{-\nu+h}\right)$ we have,

$$
\lambda_{T-1}^{*}(n)=0 \text { and } u^{T-1}(n)=e^{-2 \nu n+h n}
$$


If instead $\nu>h$ then $\lambda_{T-1}^{*}(n)=\lambda_{M}$ for all $n$. Assume that at the $t$-th step $u^{t}(n)=e^{(-(T-t+1) \nu+h) n}$. We can we write the inductive step

$$
\begin{aligned}
u^{t+1}(n)= & \min _{\lambda \in \Omega_{S}}\left(e^{-\nu n} \sum_{n \leq n^{\prime} \leq N} p_{n, n^{\prime}}^{\lambda} e^{(-(T-t+1) \nu+h) n}\right) \\
= & e^{(-\nu(T-(t+1)-1)+h) n} \\
& \left.\min _{\lambda \in \Omega_{S}}\left(q_{\lambda}+\left(1-q_{\lambda}\right) e^{(-(T-t+1) \nu+h) n}\right)\right)^{N-n}
\end{aligned}
$$

from which again the control, as at stage $T-1$, only takes extreme values. Thus the first part of the lemma is proved.

For the second part, let $t_{1}$ represent the greatest $t$ less than $t_{D c}^{*}$, i.e., $t_{1}$ is the greatest time with $\left(T-t_{1}\right) \nu>h$. Define $\chi:=e^{-\left(T-t_{1}\right) \nu+h}$ and note that $\chi<1$ and that $u^{t_{1}+1}(n)=$ $\chi^{n}$. As before, for any $n$, the following simplification results

$$
\sum_{n \leq n^{\prime} \leq N} p_{n, n^{\prime}}^{\lambda} u^{t_{1}+1}\left(n^{\prime}\right)=\chi^{n}\left(\chi+q_{\lambda}(1-\chi)\right)^{N-n} .
$$

By monotonicity of the function $\lambda \mapsto \chi+q_{\lambda}(1-\chi)$ we have, $\lambda_{t_{1}}^{*}(n)=\lambda_{M}$ and

$$
\begin{aligned}
u^{t_{1}}(n) & =\zeta^{n}\left(\chi+q_{\lambda_{M}}(1-\chi)\right)^{N} \text { for all } n \text { where } \\
\zeta & :=\frac{e^{-\nu} \chi}{\chi+q_{\lambda_{M}}(1-\chi)} .
\end{aligned}
$$

Let $t_{2}:=t_{1}-1$. Then by simplifying as before,

$$
\begin{aligned}
& \sum_{n \leq n^{\prime} \leq N} p_{n, n^{\prime}}^{\lambda} u^{t_{1}}\left(n^{\prime}\right) \\
& =\zeta^{n}\left(\chi+q_{\lambda_{M}}(1-\chi)\right)^{N}\left(\zeta+q_{\lambda}(1-\zeta)\right)^{N-n} .
\end{aligned}
$$

Clearly $\zeta<1$ and hence for all $n, \lambda_{t_{2}}^{*}(n)=\lambda_{M}$ and

$$
\begin{aligned}
u^{t_{2}}(n)=\left(\chi+q_{\lambda_{M}}(1-\chi)\right)^{N}\left(\zeta+q_{\lambda_{M}}(1-\zeta)\right)^{N} & \\
& \left(\frac{\zeta e^{-\nu}}{\zeta+q_{\lambda_{M}}(1-\zeta)}\right)^{n} .
\end{aligned}
$$

The last sentence of the lemma is proved by backward induction, using similar logic, all the way till $t=1$.

Proof of Lemma 2: Note that $\lambda_{t}^{*}(n)=$ $\arg \min _{\lambda \in \Omega_{S}} f_{T-1}(\lambda)$ with

$$
f_{T-1}(\lambda):=e^{Q_{n} \lambda^{\beta}-\lambda}\left[P_{T-1}+e^{\lambda}\right]
$$

We obtain $\lambda_{t}^{*}$ with the help of continuous space minimizer $\lambda^{*}:=\arg \min _{\lambda \in\left[0, \lambda_{M}\right]} f_{T-1}(\lambda)$ While $f_{T-1}$ is minimized over the interval $\left[0, \lambda_{M}\right]$, we note that it is a product of two functions. The first function is initially decreasing with $\lambda$ and then starts to increase while the second function is always increasing with $\lambda$. The first function at maximum can decrease till $\bar{\lambda}:=\inf _{\lambda}\left\{Q_{n} \lambda^{\beta}<\lambda\right\}$, because after this $\bar{\lambda}$, both the functions are increasing with $\lambda$. Thus, $\lambda^{*}<\bar{\lambda}$. Thus if the first non zero rate $\lambda_{1}>\bar{\lambda}$, i.e., if

$$
Q_{n} \lambda_{1}^{\beta}>\lambda_{1} \text {, then } \arg \min _{\lambda \in \Omega_{S}} f_{T-1}(\lambda)=0, u^{T-1}=e^{-2 \nu n},
$$

as in this case $f_{T-1}(0)<f_{T-1}\left(\lambda_{1}\right)$ and $f_{T-1}$ is increasing beyond $\lambda_{1}$. Therefore,

$$
\lambda_{T-1}^{*}(n)=0 \text { and } u_{T-1}(n)=e^{-2 \nu n} \text { for all } n \geq n_{o f f}(\beta, h) \text {. }
$$

For such $n$, in similar way we can write that,

$$
\begin{aligned}
u^{T-2}(n) & =e^{-\nu(n+2 N)}\left[\min _{\lambda \in \Omega} f_{T-2}(\lambda)\right]^{N-n} \text { with } \\
f_{T-2}(\lambda) & :=\left(e^{Q_{n} \lambda^{\beta}-\lambda}\left[e^{2 \nu}-1+e^{\lambda}\right]\right)
\end{aligned}
$$

Now using similar logic as before,

$\lambda_{T-2}^{*}(n)=0$ and $u_{T-2}(n)=e^{-3 \nu n}$ for all $n \geq n_{o f f}(\beta, h)$.

This continues and we have the first part of the lemma.

When $n<n_{o f f}, Q_{n} \lambda_{1}^{\beta}<\lambda_{1}$ and so from (8),

$f_{T-1}(0)>f_{T-1}\left(\lambda_{1}\right) \Longrightarrow \lambda_{T-1}^{*}(n)=\lambda_{j}$ for some $j \geq 1$.

With $o_{\lambda}^{t}(n):=e^{-\nu n+h \lambda^{\beta}} \sum_{n \leq n^{\prime} \geq n} p_{n, n^{\prime}}^{\lambda} u^{t+1}(n), u^{t}(n)=$ $\min _{\lambda \in \Omega_{S}} o_{\lambda}^{t}(n)$. The result is proved by backward mathematical induction: if it holds for some $t+1$ with $\lambda_{t+1}^{*}(n)=\lambda_{j}$ for some $j \geq 1$, then it holds at $t$, as for this $n$

$$
\begin{aligned}
o_{0}^{t}(n) & =e^{-\nu n} u^{t+1}(n)=e^{-2 \nu n+h \lambda_{j}^{\beta}} \sum_{n \leq n^{\prime} \leq N} p_{n, n^{\prime}}^{\lambda_{j}} u^{t+2}\left(n^{\prime}\right) \\
& =e^{-\nu n+h \lambda_{j}^{\beta}} \sum_{n \leq n^{\prime} \leq N} p_{n, n^{\prime}}^{\lambda_{j}} o_{\lambda_{j}}^{t+1}\left(n^{\prime}\right) \\
& \geq e^{-\nu n+h \lambda_{j}^{\beta}} \sum_{n \leq n^{\prime} \leq N} p_{n, n^{\prime}}^{\lambda_{j}} u^{t+1}\left(n^{\prime}\right)=o_{\lambda_{j}}^{t}(n),
\end{aligned}
$$

and so, $\lambda_{t}^{*}(n) \neq 0$ and hence $\lambda_{t}^{*}(n) \geq \lambda_{1}$.

\section{APPENDIX B}

\section{Contact times and propagation coefficient:}

Let $v$ be the speed of a tagged mobile with range $R$. Assume that most of the power is used to send short beacons spaced by some time interval $d t$. Case (i): velocities and ranges are such that all the mobiles in its range are likely to be different than those in the previous beacon transmission. Then the contact rate is proportional to the area covered at each transmission of a beacon, i.e. to $R^{2}$. The range needed for a given contact rate is thus of the order of square root of the rate.

Case (ii): If in contrast, the velocities are not so large, then the rate of contacts is proportional to $\pi R v d t$.

Assume we need power $p$ to be able to receive a beacon at a range $R$. If the path loss is $\alpha$ then we need to transmit at a power of $p R^{\alpha}$. Within case 2 , the contact rate is linear in $R$ so the transmission power needed behaves like $p \lambda^{\alpha}$ (where $\lambda$ is the contact rate). If the price is proportional to the power then we conclude that the price for a given contact rate is of order of $\lambda^{\alpha}$. Within case 1 , since the range is of the order of $\sqrt{\lambda}$, then the power needed to obtain $\lambda$ is of the order of $\lambda^{\alpha / 2}$. With cost linear in the power used, this is also the order of the cost for a rate of $\lambda$. 